\title{
The star ingesting luminosity of intermediate mass black holes in globular clusters
}

\author{
Enrico Ramirez-Ruiz ${ }^{1}$ and Stephan Rosswog ${ }^{2}$
}

\begin{abstract}
The dynamics of stars in the inner regions of nearby globular clusters (GCs) such as G1 indicate the presence of central concentrated dark masses, and one would like to know whether these are indeed intermediate mass black holes (IMBHs). As the number of surrounding stars, and their motions, are roughly known, the capture rate can be estimated; the question then arises of whether the apparent quiescence of the nuclei of these GCs is compatible with the IMBH's presence. The role of debris from disrupted stars in activating quiescent nuclei of GCs is studied here employing three-dimensional hydrodynamics simulations. It is argued that when individual stars are disrupted, the bulk of the debris would be swallowed or expelled rapidly compared with the interval between successive disruptions. A transient (predominantly of soft X-ray emission) signal could persist steadily with $L \sim L_{\text {Edd }}=10^{41}\left(M_{\mathrm{h}} / 10^{3} M_{\odot}\right) \mathrm{erg} / \mathrm{s}$ for at most tens of years; thereafter the flare would rapidly fade. While the infall rate declines as $t^{-5 / 3}$, some material may be stored for a longer time in an accretion disk. The IMBH luminosity could then remain as high as $L_{X} \geq 10^{39} \mathrm{erg} / \mathrm{s}$ for several hundreds of years after disruption. In a given object, this ultraluminous X-ray activity would have a duty cycle of order $10^{-4}$. Quiescent GCs, those with IMBHs now starved of fuel, should greatly outnumber active ones; observational constraints would not then be stringent until we had observed enough candidates to constitute a proper ensemble average.
\end{abstract}

Subject headings: black hole physics - hydrodynamics - globular clusters: general

\section{Introduction}

Suggestive evidence has accumulated that intermediate mass black holes exist in some globular clusters. There is some dynamical evidence for a mass concentration within the

\footnotetext{
${ }^{1}$ Department of Astronomy and Astrophysics, University of California, Santa Cruz, CA 95064

${ }^{2}$ School of Engineering and Science, Jacobs University Bremen, Campus Ring 1, 28759 Bremen, Germany
} 
central regions of some globular clusters. The dynamics of stars in the inner regions of nearby clusters such as M15, G1 and $\omega$ Centauri suggest the presence of black holes with masses of about $10^{3}, 10^{4}$ and $4 \times 10^{4} M_{\odot}$, respectively (Gerssen et al. 2002, 2003; Gebhardt et al. 2002, 2005; Pooley \& Rappaport 2006; Noyola et al. 2008). The peculiarities of an unresolved radio source in G1 indicate some unique object at the center (Ulvestad et al. 2007). Recently, somewhat arguable evidence has arisen for the presence of IMBHs in young star clusters, where ultraluminous, compact X-ray sources (ULXs) have been preferentially found to occur (Zezas et al. 2002). Their high luminosities suggest that they are IMBHs rather than binaries containing a normal stellar mass black hole (Portegies Zwart et al. 2004). But before accepting this conclusion (and dismissing alternative ideas) one would like some independent corroboration of the IMBH hypothesis, or, conversely, some way of ruling it out.

We are used to the idea that black holes are implicated in the most powerful sources in the universe, and can (when accreting) be ultra-efficient radiators. But there is, for example, no sign of such activity in M15 - the X-ray upper limit is no more than $6 \times 10^{32} \mathrm{erg} / \mathrm{s}(\mathrm{Ho}$ et al. 2003). So could a black hole be so completely starved of fuel that it does not reveal its presence? We do not directly know how much gas there is near the IMBH $(\overline{P f a h l} \&$ Rappaport 2001; Pooley \& Rappaport 2006), and there is no a priori reason why this region should be swept clean of gas. Gas that is lost from nearby stars (Baumgardt et al. 2006) as well as mass transfer from potentially bound companions (Blecha et al. 2006; Patruno et al. 2006; Hopman et al. 2004) can produce observable signatures. However, it is uncertain whether gas can be adequately supplied to explain ULX activity. The star density, on the other hand, is much better known - after all, if the stars were not closely packed near the center of the cluster we would not have evidence for the black hole at all. As stellar orbits diffuse in phase space, it therefore seems inevitable that some may wander sufficiently close to the hole that they suffer tidal disruption. When a star is disrupted, there is bound to be some radiation from the sudden release of gas. The flares resulting from a disrupted star could be the clearest diagnostic of a IMBH's presence.

It is an intricate although tractable problem in stellar dynamics to calculate the chance that a star passes within the IMBH's tidal radius (Frank \& Rees 1976). In a simple case when the velocities are isotropic, the frequency with which a star would enter the zone of vulnerability is $\xi \sim 10^{-7} M_{\mathrm{h}, 3}^{4 / 3}\left(n_{*} / 10^{6} \mathrm{pc}^{-3}\right)\left(\sigma / 10 \mathrm{~km} \mathrm{~s}^{-1}\right)^{-1} \mathrm{yr}^{-1}$, where $n_{*}$ is the stellar number density in the cluster nucleus and $M_{\mathrm{h}, 3}$ is the black hole's mass in units of $10^{3} M_{\odot}$. This estimate although simplified, agrees well with the fiducial rates derived from detailed $N$-body simulations of multi-mass star clusters containing IMBHs (Baumgardt et al. 2004). It may seem, however, that even the modest rate of stellar disruptions given above could have conspicuous consequences. The debris from a disrupted one solar-mass star per hundred 
million years, swallowed steadily with 10 per cent radiative efficiency, would yield a luminosity of $L_{\text {steady }} \sim 6 \times 10^{37} \mathrm{erg} / \mathrm{s}$ - higher than is observed for X-ray binaries in quiescence. But in reality, one expects brighter flares with short duty cycles, as the time it takes to digest or expel the debris from one star is much shorter than the mean interval between one stellar disruption and the next. This Letter is concerned with the observational manifestations of such phenomena, with particular reference to globular clusters where the masses of the black holes (if indeed present) are perhaps of order $10^{3}-10^{4} M_{\odot}$.

\section{The tidal Disruption of solar-type star by an IMBH}

A star interacting with a massive black hole cannot be treated as a point mass if it gets so close to the hole that it becomes vulnerable to tidal distortions. Such effects become important when the pericenter distance becomes as small as the tidal radius: $R_{\mathrm{T}} \simeq 5 \times$ $10^{11} M_{\mathrm{h}, 3}^{1 / 3}\left(R_{*} / R_{\odot}\right)\left(M_{*} / M_{\odot}\right)^{-1 / 3} \mathrm{~cm}$. The gravitational radius, $R_{\mathrm{g}} \simeq 1.5 \times 10^{8} M_{\mathrm{h}, 3} \mathrm{~cm}$, scales with mass, whereas the tidal radius goes only as the cube root. The tidal forces at their "surfaces" are thus more gentle for black holes of larger mass, and solar-type stars would be disrupted only after passing irreversibly inside an ultra-massive hole's horizon: $M_{\mathrm{h}} \leq$ $7 \times 10^{7} M_{\odot}$.

When a rapidly changing tidal force starts to compete with a star's self-gravity, the material of the star responds in a complicated way, being stretched along the orbital direction and squeezed at right angles to the orbit Carter \& Luminet 1983; Rees 1988; Evans \& Kochanek 1989). To study this problem, we use a three dimensional smoothed particle hydrodynamics method (SPH) to solve the equations of hydrodynamics. Due to its Lagrangian nature SPH is perfectly suited to follow tidal disruption processes during which the corresponding geometry, densities and time scales are changing violently. The SPH-formulation that we use in this study is described in Rosswog et al. (2008a b). Common to all runs is the initiation of the calculations with the star being place safely outside $R_{\mathrm{T}}$ and set it onto a parabolic orbit so that $R_{\text {min }}=R_{\mathrm{T}} / 3$ for a $10^{3} M_{\odot}$ black hole. We have considered three different initial conditions for the approaching star, constructed here by solving the spherically symmetric Lane-Emden equations: A $[1,1,0.6] M_{\odot}$ solar-type star modeled with a polytropic equation of state with adiabatic index $\Gamma=[5 / 3,1.4,1.4]$ and $R_{*}=[1,1,0.75] R_{\odot}$.

The gross quantitative behavior of a solar-type star plunging deeply within the tidal radius is examined here (the reader is refer to $\$ 3$ for a discussion on the role of the stellar density structure in shaping the history of the mass accretion rate). Several snapshots taken from our numerical simulations of a $1 M_{\odot}$ solar-type star (modeled with $\Gamma=5 / 3$ ) are shown in Figure 1. The tidal bulge raised on the star by the black hole becomes of 
an order unity distortion near pericenter. The resultant gravitational torque spins it up to a good fraction of its corotation angular velocity by the time it gets disrupted. This takes place on a timescale comparable to the crossing time of the star through periastron, $\Delta t \sim R_{*} / v_{\mathrm{p}} \sim 349.6\left(M_{*} / M_{\odot}\right)^{-1 / 6}\left(R_{*} / R_{\odot}\right)^{3 / 2} M_{\mathrm{h}, 3}^{-1 / 3} \mathrm{~s}$.

The energy required to tear the star apart (that is the star's self binding energy) is of order $M_{*} v_{*}^{2}$, where $v_{*}=\left(G M_{*} / R_{*}\right)^{1 / 2}$. During tidal disruption, this energy is supplied at the expense of the orbital kinetic energy, which at pericenter $\sim R_{\mathrm{T}}$ is larger by $\sim\left(M_{\mathrm{h}} / M_{*}\right)^{2 / 3}$ (Rees 1988). Figure 2 displays the evolution of the differential mass distribution in specific energy for the debris. Disruption reduces the orbital energy by the binding energy of the star $\epsilon_{*} \sim\left(G M_{*} / R_{*}\right) \approx 10^{-5} c^{2}$, which is much smaller than the specific kinetic energy at pericenter. The variation of the specific energy in the released gas is determined mainly by the relative depth of a mass element across the disrupted star in the potential well of the black hole. The spread in this specific energy is of order $v \Delta v \sim 10^{-4} M_{\mathrm{h}, 3}^{1 / 3} c^{2}$, where $v \approx v_{\mathrm{p}}=c\left[R_{\mathrm{g}} / R_{\mathrm{T}}\right]^{1 / 2}$ and $\Delta v \approx v_{*}=\left[G M_{*} / R_{*}\right]^{1 / 2}$. This is much larger than $\epsilon_{*}$ and, as a result, almost half the debris escapes on hyperbolic orbits with speeds $\sim 3000 M_{\mathrm{h}, 3}^{1 / 6} \mathrm{~km} \mathrm{~s}^{-1}$; the kinetic energy output being $\sim 10^{50} M_{\mathrm{h}, 3}^{1 / 3}$ erg (comparable to the energy of a supernova). The material would be concentrated in a fan close to the orbital plane. Adiabatic cooling, as the material expands, severely reduces the internal radiative energy content before the debris became optically thin. The escaping radiation from the unbound debris would therefore release much less than the initial energy content of the star. There would therefore be no conspicuous flare, until the bound debris fell back onto the IMBH.

\section{Ingesting the Stellar Debris}

The returning gas does not immediately produce a flare of activity from the black hole. First material must enter quasi-circular orbits and form an accretion torus (Evans \& Kochanek 1989). Only then will viscous effects release enough binding energy to power a flare. The bound orbits are very eccentric, and the range of orbital periods is large. The orbital semi-major axis of the most tightly bound debris is $a \sim 10^{4} M_{\mathrm{h}, 3}^{-1 / 3}\left(R_{*} / R_{\odot}\right)\left(M_{*} / M_{\odot}\right)^{-2 / 3} R_{\mathrm{g}}$, and the period is only $t_{\mathrm{a}} \sim 6300\left(a / 1.5 \times 10^{4} R_{\mathrm{g}}\right)^{3 / 2} M_{\mathrm{h}, 3}^{-1 / 2} \mathrm{~s}$. If the gaseous debris suffered no internal dissipation due to high viscosity or shocks, it would, after one or two orbital periods, form a highly elliptical disk with a big spread in apocentric distances between the most and least bound orbits, but where at pericenter the orbits are all squeezed in a range $\delta R / R \sim R_{*} / R_{\tau}=0.1\left(M_{*} / M_{\mathrm{h}, 3}\right)^{1 / 3}$. As the stream approaches pericenter, the radial focusing of the orbits therefore acts as an effective nozzle (Figure 3). After pericenter passage, the outflowing gas is on orbits which collide with the infalling stream near the original orbital 
plane at apocenter, giving rise to an angular momentum redistributing shock (Figure 3) much like those in cataclysmic variable systems. The debris raining down would, after little more than its free-fall time, settle into a disk.

This orbiting debris starts forming a disk when the most highly bound debris falls back. The simulation shows that the first material returns at a time $\leq t_{\mathrm{a}}$, with a peak infall rate roughly given by $31 M_{\odot} \mathrm{yr}^{-1}$ (Figure 4). Such high infall rates are expected to persist, relative steadily, for at least a few orbital periods, before all the highly bound material rains down. Since the amount of highly bound material $(d M / d \epsilon)$ depends sensitively on the density structure of the star, we find that more centrally condensed stars produce mass feeding rates that are slightly larger in comparison to less centrally condensed stars of comparable mass although they reach the self-similar $\propto t^{-5 / 3}$ phase after fewer orbital periods. The vicinity of the hole would thereafter be fed solely by injection of the infalling matter at a rat $\mathrm{C}^{1}$ that drops off roughly as $t^{-5 / 3}$ for $t \geq t_{\mathrm{fb}} \approx 10^{5} \mathrm{~s}$. Once the torus is formed, it will evolve under the influence of viscosity, radiative cooling winds and time dependent mass inflow.

The black hole cannot accept matter, with a radiative efficiency $\epsilon=0.1 \epsilon_{0.1}$, at a rate exceeding $\dot{M}_{\text {Edd }} \approx 2 \times 10^{-5} \epsilon_{0.1} M_{\mathrm{h}, 3} M_{\odot} \mathrm{yr}^{-1}$, without exceeding the black hole's Eddington luminosity. The rate at which the stellar debris returns to the vicinity of the black hole exceeds this limit: $\dot{M} \approx 10^{6} \dot{M}_{\text {Edd }}$ for $\epsilon=0.1$. Given that this high rate can only be sustained by infall of the highly bound material for a time $t_{\mathrm{fb}}$, we infer that, if the viscosity were high enough to process all the material within the infall timescale, then the luminosity of the hole could not remain as high as the Eddington luminosity for longer than $t_{\mathrm{Edd}} \approx$ $5 \times 10^{3} \epsilon_{0.1}^{-3 / 5} M_{\mathrm{h}, 3}^{-3 / 5} t_{\mathrm{fb}} \sim 17 \epsilon_{0.1}^{-3 / 5} M_{\mathrm{h}, 3}^{-3 / 5} \mathrm{yr}$. The viscosity would have to be implausibly low (i.e. the usual viscous dissipation time $t_{\mathrm{d}}$ for a thick disk would be $\alpha^{-1} ; \alpha$ would have to be below $10^{-5}$ for $t_{\mathrm{d}} \geq t_{\text {Edd }}$ ) for the bulk of the mass to be stored for longer than $t_{\text {Edd }}$ in a reservoir at $R \sim R_{\mathrm{T}}$. A luminosity $\sim L_{\mathrm{Edd}}=10^{41} M_{\mathrm{h}, 3} \mathrm{erg} \mathrm{s}^{-1}$ can therefore only be maintained for at most ten years; thereafter the flare would continue to fade as $t^{-3 / 5}$. It is clear from the behavior of $\dot{M}$ that most of the debris would be fed to the hole far more rapidly than it could be accepted if the radiative efficiency were high; much of the bound debris must either escape in a radiatively-driven outflow or be swallowed inefficiently.

\footnotetext{
${ }^{1}$ Note that the rate at which mass returns to the black hole is only proportional to $t^{-5 / 3}$ when $d M / d \epsilon$ is constant (Rees 1988; Phinney 1989). Figure 2 displays the differential mass distributions in specific energy obtained by the simulation, which show that $d M / d \epsilon$ is nearly constant.
} 


\section{Discussion}

\subsection{Observability of $L_{\mathrm{Edd}}$ Flares}

A distinctive consequence of a $10^{3}-10^{4} M_{\odot}$ IMBH's presence in the centers of globular clusters would be the transient flares produced as the bound debris from the disrupted solar-type stars is swallowed, the luminosities being as high as $L_{\mathrm{Edd}}=10^{41} M_{\mathrm{h}, 3} \mathrm{erg} \mathrm{s}^{-1}$. The rise and the peak bolometric luminosity can be predicted with some confidence. However, the effective surface temperature (and thus the fraction of luminosity that emerges predominantly in the soft X-ray band) is harder to predict, as it depends on the size of the effective photosphere that shrouds the hole. For $t \lesssim t_{\text {Edd }}$, the effective surface temperature should be $T \lesssim T_{\mathrm{Edd}}=\left(L_{\mathrm{Edd}} / 4 \pi \sigma R_{\mathrm{g}}^{2}\right)^{1 / 4} \sim 1 M_{\mathrm{h} .3}^{-1 / 4} \mathrm{keV}$. In a given globular cluster, these flares would have a duty cycle of order $\xi t_{\mathrm{Edd}} \sim 10^{-6}\left(\xi / 10^{-7} \mathrm{yr}^{-1}\right)\left(t_{\mathrm{Edd}} / 10 \mathrm{yr}\right)$, and as a result, quiescent GCs, those with IMBHs now starved of fuel, should greatly outnumber active ones.

Given a globular cluster space density of $n_{\mathrm{gc}} \sim 4 \mathrm{Mpc}^{-3}$ (Brodie \& Strader 2006), we expect the density rate of flares with $L=L_{\text {Edd }}$ to be at most $\sim 4000 \mathrm{yr}^{-1} \mathrm{Gpc}^{-3}$, if $10^{3}-10^{4} M_{\odot}$ black holes were prevalent in globular clusters. Therefore we would not yet expect to have detected such a flare. Observational constraints on the presence of IMBH would not then be stringent until a sufficiently large sample of galaxies should be sampled in order to reveal some of their GC members in a flaring state. Such objects should be searched for out to large distances. However, assuming $L \sim 10^{41} \mathrm{erg} \mathrm{s}^{-1}$ and $T \sim T_{\text {Edd }}$, we find that EXIST (Grindlay 2004) would only be able to detect such a flaring event out to a distance of about $1 \mathrm{Mpc}$.

\subsection{ULX activity}

A further question is how fast the luminosity fades after the flare. This is important because we want to know whether the IMBH has faded below ULX levels before the next stellar disruption occurs. The answer to this question depends on how long it takes the last residues of the star to be ingested. One expects the infall rate $\dot{M}$ to decline as $t^{-5 / 3}$ for late times. Some material may, however, be stored for a longer time in an accretion disk. This is mainly because the specific angular momentum for Keplerian orbits grows $\propto r^{1 / 2}$, so angular momentum transport via disk viscosity requires that $10 \%$ of the debris goes out to $10^{3} R_{\mathrm{T}}$ and $1 \%$ to $10^{5} R_{\mathrm{T}}$ before being swallowed.

The evolution of the bound debris onto a black hole for $t \geq t_{\mathrm{a}}$ has been studied by Cannizzo et al. (1990) using a time dependent $\alpha$-disk model. Cannizzo and collaborators 
concluded that the black hole's luminosity fades more slowly: $L \propto t^{-1.2}$ and depends only weakly on the disk viscosity. The IMBH luminosity could then remain as high as $L_{X} \geq$ $10^{39} \mathrm{erg} \mathrm{s}^{-1}$ for several hundreds of years after disruption. In a given object, this ULX activity would have a duty cycle of order $10^{-4}$ unless the rate of disruptions is much larger than $10^{-7}$ or the viscosity were so low that the bulk of the mass could be stored for many thousands of years at $R \sim R_{\mathrm{T}}$ (where the dynamical timescale is only a few hours).

\subsection{Relevance to GCs and IMBH Growth}

The mass fraction that is ejected rather than ingested, although less spectacular than the accretion-powered flares could nonetheless have an important influence on the energy balance within a GC - similar than a supernova exploding in the same volume. When the star is disrupted in a single flyby, about half the debris is ejected in a fast moving spray of gas; the kinetic energy output being $\sim 10^{50} M_{\mathrm{h}, 3}^{1 / 3} \mathrm{erg}$. The ejecta would be braked and their kinetic energy thermalized, as they ran into the diffuse gas (probably originating from stellar mass loss). If this material is effectively shock heated, it could contribute to the X-ray and radio luminosity of the GC long after the star has been disrupted. Stellar disruption may have other implications - for instance, to the dynamics of line emitting regions within GCs (Chomiuk et al. 2008).

The most tightly bound debris, on the other hand, would traverse an elliptical orbit before returning to $R_{\mathrm{T}}$. There would therefore be no conspicuous flare until, as discussed above, the bound debris fall back into the hole. Electron scattering opacity almost certainly dominates the radiative transfer and the photons will be trapped out to a radius $R_{\tau}=$ $\dot{M} \kappa_{\tau} / 4 \pi c$. Energy will be dissipated at a supercritical rate as the material swirls closer to the hole, some gas may then be ejected in a radiation-driven wind. In principle it is possible for only a fraction $\epsilon^{-1}\left(R_{\mathrm{g}} / R_{\mathrm{T}}\right) \sim 0.004 \epsilon_{0.1}\left(R_{*} / R_{\odot}\right)^{-1}\left(M_{*} / M_{\odot}\right)^{1 / 3} M_{\mathrm{h}, 3}^{2 / 3}$ to be actually swallowed, all the remainder being ejected. If a fraction $f$ of the mass from disrupted stars is accreted onto the black hole, the average rate of tidal disruptions required to form an IMBH out of $\mathrm{a} \sim 50 M_{\odot}$ progenitor would need to be $\geq 10^{-6} M_{\mathrm{h}, 3}(f / 0.1)^{-1}\left(\tau_{\mathrm{GC}} / 10^{10} \mathrm{yr}^{-1} \mathrm{yr}^{-1}\right.$, where $\tau_{\mathrm{GC}}$ is the globular cluster age. In such cases, the density would, however, be high enough that runaway merging of high-mass main-sequence stars could lead directly to the formation of an IMBH (Baumgardt et al. 2006; Portegies Zwart et al. 2004).

We thank H. Baumgardt, L. Chomiuk, J. Strader, J. Guillochon, P. Hut, E. Noyola, C. Hopman, J. Kalirai, D. Kasen and M. Rees for useful discussions and the referee for constructive suggestions. E. R. acknowledges support from the DOE (SciDAC; DE-FC02- 
01ER41176) and NSF (0521566). The simulations presented in this paper were performed on the JUMP computer of the Höchstleistungsrechenzentrum Jülich.

\section{REFERENCES}

Baumgardt, H., Makino, J., \& Ebisuzaki, T. 2004, ApJ, 613, 1143

Baumgardt, H., Hopman, C., Portegies Zwart, S., \& Makino, J. 2006, MNRAS, 372, 467

Blecha, L., Ivanova, N., Kalogera, V., Belczynski, K., Fregeau, J., \& Rasio, F. 2006, ApJ, 642,427

Brodie, J. P., \& Strader, J. 2006, ARA\&A, 44, 193

Cannizzo, J. K., Lee, H. M., \& Goodman, J. 1990, ApJ, 351, 38

Carter, B., \& Luminet, J.-P. 1983, A\&A, 121, 97

Chomiuk, L., Strader, J., \& Brodie, J. P. 2008, AJ, 136, 234

Evans, C. R., \& Kochanek, C. S. 1989, ApJ, 346, L13

Frank, J., \& Rees, M. J. 1976, MNRAS, 176633

Gebhardt, K., Rich, R. M., \& Ho, L. C. 2002, ApJ, 578 L41

Gebhardt, K., Rich, R. M., \& Ho, L. C. 2005, ApJ, 634, 1093

Gerssen, J., et al. 2002, AJ, 124, 124, 3270

Gerssen, J., et al. 2003, AJ, 125, 376

Grindlay, J. E. 2004, X-ray Timing 2003: Rossi and Beyond, 714, 413

Ho, L. C., Terashima, Y., \& Okajima, T. 2003, ApJ, 587, L35

Hopman, C., Portegies Zwart, S. F., \& Alexander, T. 2004, ApJ, 604, L101

Noyola, E., Gebhardt, K., \& Bergmann, M. 2008, ApJ, 676, 1008

Patruno, A., Portegies Zwart, S., Dewi, J., \& Hopman, C. 2006, MNRAS, 370, L6

Pfahl, E., \& Rappaport, S. 2001, ApJ, 550, 172

Phinney, E.S. 1989, IAU Symp. 136, 543 
Pooley, D., \& Rappaport, S. 2006, ApJ, 644, L45

Portegies Zwart, S. F., et al. 2004, Nature, 428, 724

Rees, M. J. 1988, Nature, 333, 523

Rosswog, S., Ramirez-Ruiz, E., Hix, W. R., \& Dan, M. 2008, Computer Physics Communications, 179,184

Rosswog, S., Ramirez-Ruiz, E., \& Hix, W. R. 2008, ApJ, 679, 1385

Ulvestad, J. S., Greene, J. E., \& Ho, L. C. 2007, ApJ, 661, L151

Zezas, A., et al. 2002, ApJ, 577, 710

This preprint was prepared with the AAS LATEX macros v5.2. 


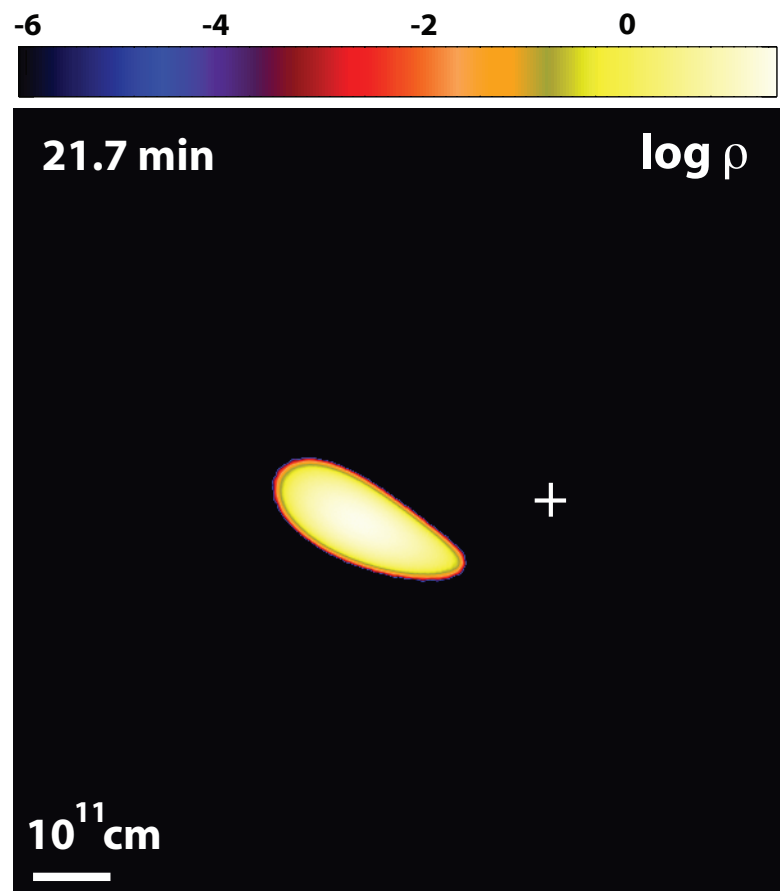

\begin{tabular}{lllllll}
-5 & -4 & -3 & -2 & -1 & 0 & 1 \\
\hline
\end{tabular}
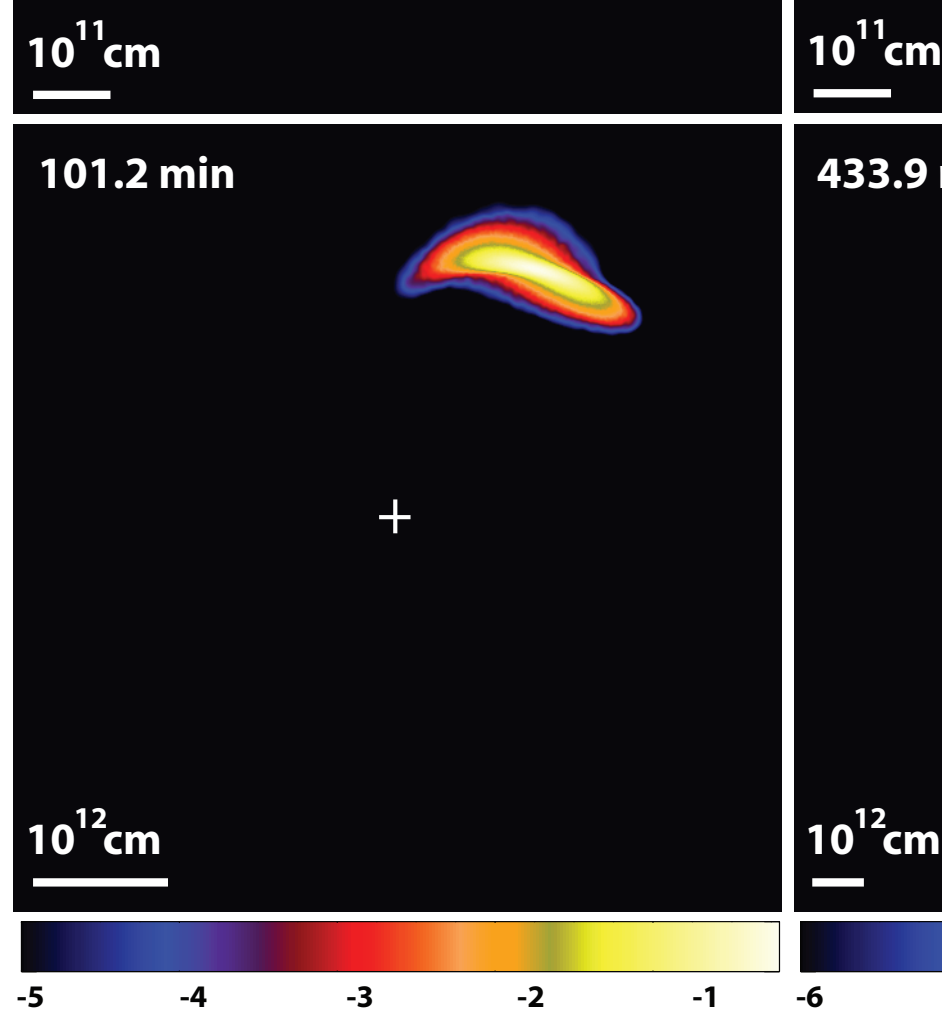

\section{$28.9 \mathrm{~min}$}

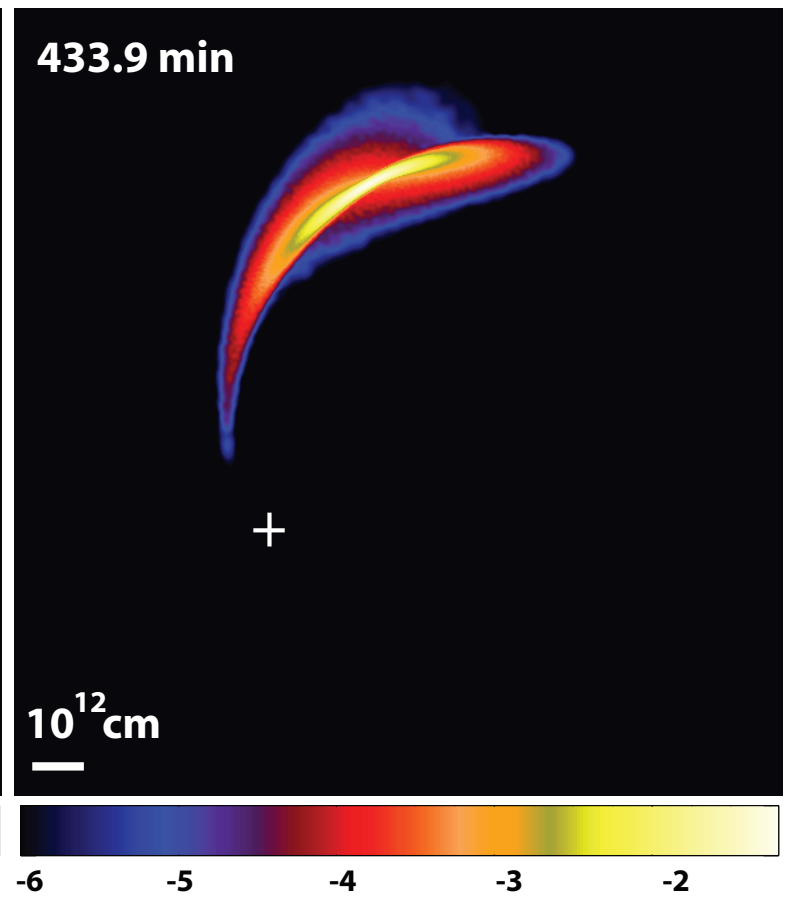

Fig. 1.- A $1 M_{\odot}$ solar-type star (modeled with more than $10^{6} \mathrm{SPH}$ particles) approaching a $10^{3} M_{\odot}$ black hole on a parabolic orbit with pericenter distance $R_{\min }=R_{\mathrm{T}} / 3$ is distorted, spun up during infall and then tidally disrupted. The panels show density cuts (in cgs units) through the orbital (xy-) plane before and after passage through pericenter. 


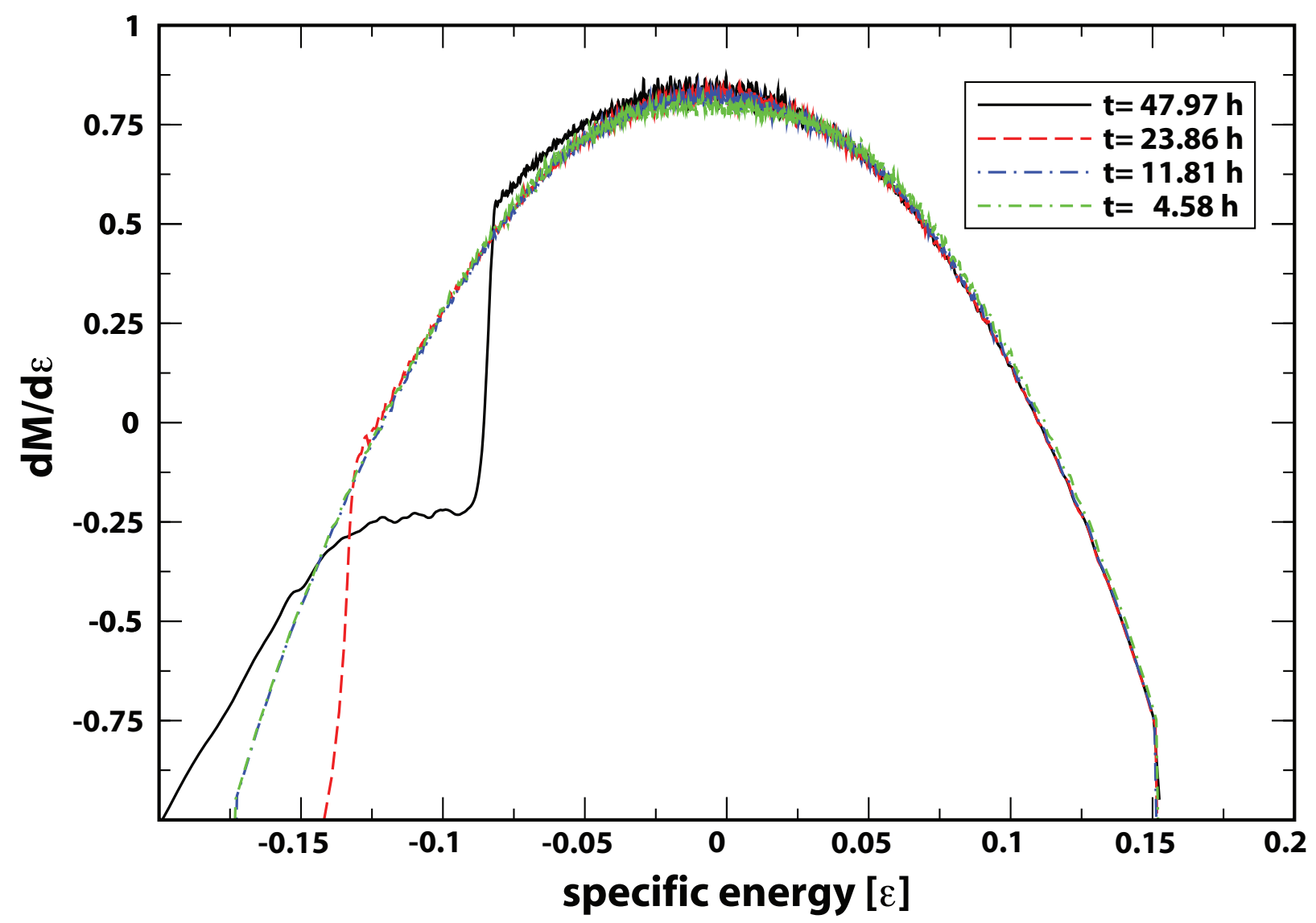

Fig. 2.- Differential mass distributions in specific energy for the $1 M_{\odot}$ stellar debris. At $t=47.97 \mathrm{~h}$, the amount of material unbound is $\sim 47 \%$ of the initial mass of the star. 


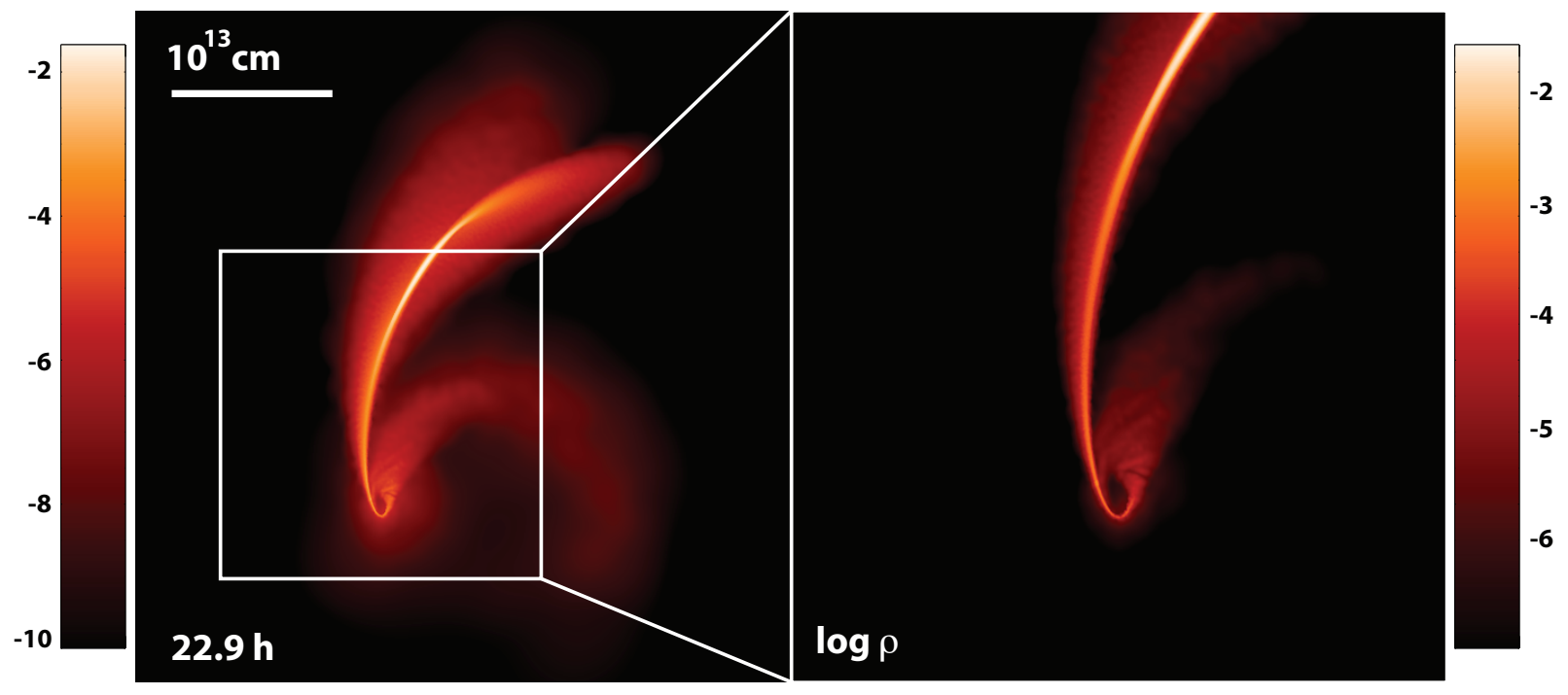

Fig. 3.- Density cuts (in cgs units) in the orbital plane of the bound debris 22.9 hours after disruption. The most tightly bound debris would transverse an elliptical orbit with major axis $\sim 10^{4} R_{\mathrm{g}}$ before returning to $R \approx R_{\mathrm{p}}$, where radial focusing of orbits acts as an effective nozzle. These orbits are focused back into the original orbital plane at pericenter. This causes the formation of a pancake shock that weakly redistributes the orbital parameters and damps out some of the vertical motion. 


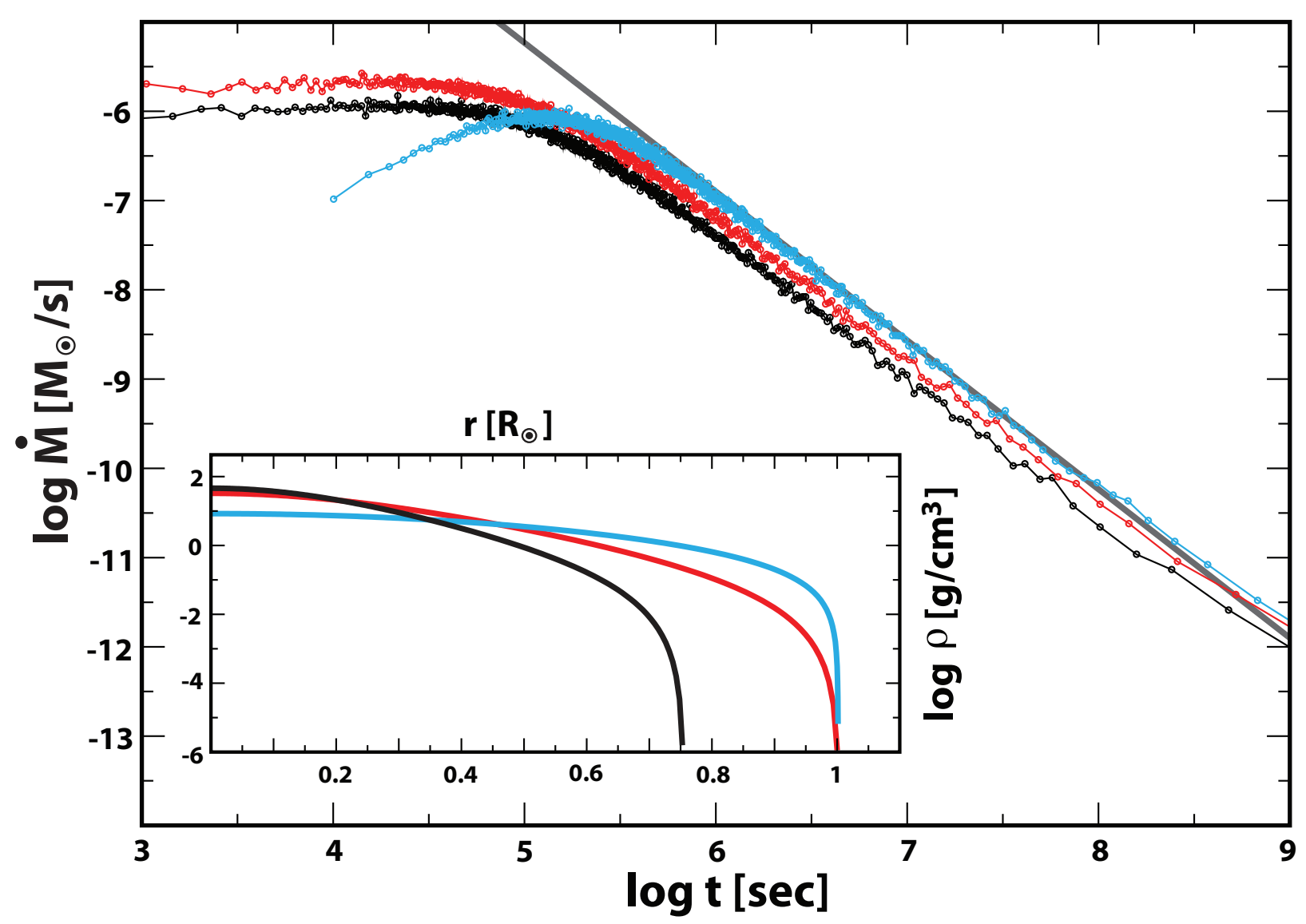

Fig. 4. - The rates at which the stellar debris returns to the vicinity of the black hole for different types of stars: A $[1,1,0.6] M_{\odot}$ solar-type star modeled with $\Gamma=[5 / 3,1.4,1.4]$ shown as a solid [blue, red, black] line. The infalling matter at a rate that drops off roughly as $t^{-5 / 3}$ (gray line). Inset: the internal density structure of the various stars. 